\title{
The Continuous Tabu Search as an Optimizer for 2D-to-3D Biomedical Image Registration
}

\author{
Mark P. Wachowiak and Adel S. Elmaghraby \\ Department of Computer Engineering and Computer Science \\ University of Louisville, Louisville, KY 40292, USA \\ \{mpwach01, aselma01\}@athena.louisville.edu
}

\begin{abstract}
Both stochastic and direct optimization of similarity metrics have been used in biomedical image registration. This paper proposes an adapted tabu search for registration of $2 \mathrm{D}$ ultrasound scans to $3 \mathrm{D}$ volumes. Accuracy and efficiency compare favorably with other methods.
\end{abstract}

\section{Continuous Tabu Search}

Stochastic global optimizers and stochastic-direct hybrids, which include genetic algorithms and simulated annealing, are used to locate the global optima of complicated and multiextremal functions. These methods have proven effective for biomedical image registration 1], [2]. In this paper, another global optimizer, the continuous tabu search (CTS) (described in [3]), is adapted to image registration.

In the adapted method, after the CTS has identified a number of "promising points" where the optimum may lie, the "affine shaker" (AS) algorithm [4] is applied to locate the best point in each promising area. The shape of the promising area is thus adapted to include areas of the search space that may have been missed during diversification. Finally, an intensified search is conducted around the most promising point using the Nelder-Mead simplex algorithm.

\section{Methods and Results}

The maximum value of the overlap-invariant normalized mutual information [5] functional was sought. Two $100 \times 100$ - and one $64 \times 64$-pixel images (three experiments) were obtained from a $148 \times 160 \times 141$-voxel US volume of a heart (courtesy of TomTec, Inc., Germany). Each experiment consisted of 4 trials for each of 10 initial points at varying distances from a reference ("true" point).

Optimizers were then used to register the $2 \mathrm{D}$ slices within the original $3 \mathrm{D}$ volume: (1) ASA(0) - Adaptive simulated annealing with slow cooling schedule [6]; (2) ASA(1) - fast cooling; (3) ASA(2) - "medium" cooling; (4) A modified Nelder-Mead simplex algorithm: All reflections of the initial solution in $6 \mathrm{D}$ space are examined to prevent finding a local optimum; (5) Tabu / AS, using discretization of the search space [7], AS, and the simplex algorithm for intensification; (6) $\mathrm{Tabu}^{\prime} / \mathrm{AS}$, as (5) above, but restarting the search with a reflected point 


\begin{tabular}{|c|c|c|c|c|c|c|c|}
\hline \multicolumn{2}{|l|}{ Experiment } & $\overline{\mathrm{ASA}(0)}$ & $\mathrm{ASA}(1)$ & $\mathrm{ASA}(2)$ & Amoeba & Tabu/AS & $\mathrm{Tabu}^{\prime} / \mathrm{AS}$ \\
\hline \multirow{3}{*}{$\begin{array}{c}\text { \% Correct } \\
\text { Reg. }\end{array}$} & 1 & $\overline{7.50}$ & 27.50 & $\overline{7.50}$ & 30 & 40 & 37.50 \\
\hline & 2 & 2.50 & 15 & 5 & 0 & 5 & 10 \\
\hline & $J$ & 5 & 30 & 5 & 10 & 42.5 & 52.5 \\
\hline \multicolumn{2}{|l|}{ Average } & 4.17 & 24.17 & 5.83 & 13.33 & 29.17 & 33.33 \\
\hline \multirow{3}{*}{$\begin{array}{c}\text { Mean Func. } \\
\text { Evals. }\end{array}$} & 1 & $6.19 \pm 0.09$ & $6.17 \pm 0.05$ & $6.34 \pm 0.07$ & $9.03 \pm 0.27$ & $4.68 \pm 0.46$ & $4.75 \pm 0.25$ \\
\hline & 2 & $6.41 \pm 0.00$ & $6.13 \pm 0.03$ & $6.22 \pm 0.06$ & $\mathrm{~N} / \mathrm{A}$ & $4.30 \pm 0.18$ & $4.34 \pm 0.51$ \\
\hline & 3 & $6.23 \pm 0.99$ & $6.18 \pm 0.08$ & $6.33 \pm 0.12$ & $9.18 \pm 0.00$ & $4.98 \pm 0.28$ & $5.00 \pm 0.25$ \\
\hline \multicolumn{2}{|l|}{ Average } & 6.28 & 6.16 & 6.30 & 9.11 & 4.66 & 4.70 \\
\hline
\end{tabular}

Table 1. Percentage of correct registrations and mean number of function evaluation for correct $2 \mathrm{D}-3 \mathrm{D}$ registration $(\times 1000)$.

after 20 iterations without improvement. The number of correct registrations and mean number of function evaluations are shown in Table 1.

The absence of feature extraction and pre-processing, no user interaction, and the large search space contributed to the low percentage of correct registration for all methods. The two CTS techniques correctly registered a larger percentage of trials. ASA(1) also performed very well. CTS outperformed ASA or the simplex in function evaluations for correct registrations. The maximum number of ASA iterations without the simplex stage (here, 4000) was often reached, indicating that CTS may explore the search space in fewer iterations (maximum 200).

Although existing direct and stochastic methods are often effective in image registration, the continuous tabu search can also be used as an optimizer for 2D-to-3D registration, and further work on this technique is warranted.

\section{References}

1. Matsopoulos, G. K., Mouravliansky, N. A., Delibasis, K. L., Konstantina, S. N.: Automatic Retinal Image Registration Scheme Using Global Optimization Techniques. IEEE Trans. Info. Tech. in Biomed. 3 (1999) 47-60.

2. Rouet, J.-M., Jacq, J.-J., Roux, C.: Genetic Algorithms for a Robust 3-D MR-CT Registration. IEEE Trans. Info. Tech. in Biomed. 4 (2000) 126-136.

3. Chelouah, R., Siarry, P.: Tabu Search Applied to Global Optimization. Euro. J. of Op. Res. 123 (2000) 256-270.

4. Battiti, R., Tecchiolli, G.: The Continuous Reactive Tabu Search: Blending Combinatorial Optimization And Stochastic Search For Global Optimization. Ann. of Op. Res. 63 (1996) 153-188.

5. Studholme, C., Hill, D. L. G., Hawkes, D. J.: An Overlap Invariant Entropy Measure of 3D Medical Image Alignment. Patt. Recog., 32 (1999) 71-86.

6. Ingber, L., Rosen, B.: Genetic Algorithms and Very Fast Simulated Reannealing: A Comparison. Math. and Computer Model. 16 (1992) 87-100.

7. Siarry, P., Berthiau, G.: Fitting of Tabu Search To Optimize Functions of Continuous Variables. Int. J. for Num. Methods in Eng. 40 (1997) 2449-2457. 